\title{
Fibroma digital infantil: presentación de un caso
}

\section{Infant digital fibroma: case report}

\author{
Nery Romero Rojas \\ Médico patólogo, Hospital San Bartolomé, Lima, Perú.
}

\begin{abstract}
Resumen
El fibroma digital infantil o fibroma por cuerpos de inclusión es una entidad de rara incidencia, que se caracteriza por una proliferación benigna de células miofibroblásticas que forman nódulos en el dorso de los dedos de manos o pies de los niños. Tiene una imagen histológica muy característica -los cuerpos de inclusión citoplasmática- y una tendencia a la recurrencia; algunos pueden regresionar espontáneamente sin tratamiento. Se presenta el caso de un niño de 4 meses de edad, que desde el nacimiento presentó un tumor en el dorso de la falange distal del tercer dedo del pie derecho. Se extirpó el tumor de 1,5 × 1,3 × 0,6 cm que a la histología mostró una proliferación de haces entrelazados de células fusiformes con núcleos vesiculosos, en cuyo citoplasma presentaba cuerpos de inclusión eosinofilicos teñidos con la coloración tricrómica de Masson y que a la inmunohistoquímica se tiñeron con vimentina, actina y desmina.

Palabras clave: Fibromatosis, digital, infantil, fibromatosis por cuerpos de inclusión, tumor fibroso digital recidivante de la infancia, miofibroblastos.
\end{abstract}

\begin{abstract}
Infant digital fibroma or inclusion body fibromatosis is a rare disorder consisting in a benign proliferative process of myofibroblast cells that in histopathology show cytoplasm inclusion bodies. Nodules develop mainly in fingers and toes of infants. There is high recurrence but spontaneous regression without treatment has also been reported. We present the case of a 4 month-old boy showing a $1.5 \times 1.3$ $x 0.6 \mathrm{~cm}$ nodule in the third finger of the right hand. Histological examination revealed spindled tumor cells containing characteristic intracytoplasmic inclusion bodies that stained with vimentin, muscle specific actin and desmin.
\end{abstract}

Keywords: Fibromatosis, digital, infant, inclusion bodies fibromatosis, infancy recurrent fibrous digital tumor, miofibroblasts.

An Fac med. 2011;72(1):79-1

\section{INTRODUCCIÓN}

La fibromatosis digital infantil o fibromatosis por cuerpos de inclusión, también llamado tumor de Reye, es un proceso proliferativo benigno de células miofibroblásticas demostradas por microscopía electrónica e inmunohistoquimica $^{(1)}$. Se presenta en infantes, se localiza principalmente en la parte dorsal y distal de las falanges de los dedos de manos y pies y tiene una importante tendencia a la recurrencia; adopta la forma de nódulos bien circunscritos, de consistencia blanda. El aspecto más distintivo y patognomónico es la presencia en el citoplasma de los mio- fibroblastos de cuerpos de inclusión redondeados eosinofílicos, de posición paranuclear ${ }^{(1-4)}$.

Presentamos un caso de origen congénito que además tenía asociado un frenillo sublingual.

\section{PRESENTACIÓN DEL CASO}

Se trata de un niño de 4 meses de edad que presentó una tumoración a nivel del dorso de la tercera falange del tercer dedo del pie derecho. Dicha tumoración la tenía desde el nacimiento y había crecido rápidamente. Era blanda, rosada, sin signos de flogosis. Se extirpó la tumoración, que midió 1,5 x 1,3 x 0,6 $\mathrm{cm}$ (figura 1), de consistencia blanda y fue procesada con la técnica convencional de hematoxilina eosina.

A la histología (figura 2) se observó haces de tejido formados por células fusiformes (figura 3), con núcleo ovalado e inclusiones intracitoplasmáticas de color rojizo. Se realizó coloración tricrómica de Masson (figura 4), que identifica las células miofibroblásticas y tiñe los cuerpos de inclusión. Se realizó las pruebas de inmunohistoquimica con desmina, actina, S100 y vimentina, saliendo positiva a vimentina (figura 5), actina y desmina (figura 6). 


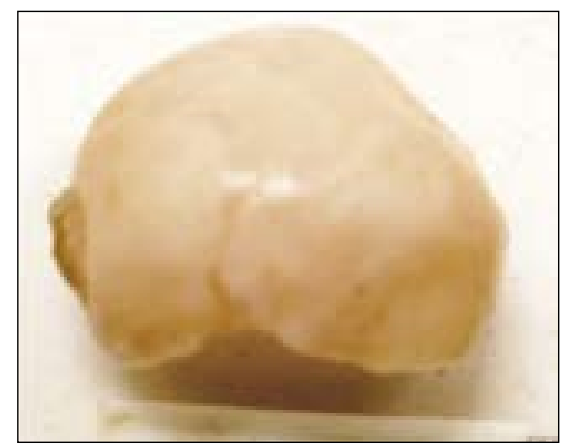

Figura 1. Tumor de Reye, vista macroscópica.

El niño nació por cesárea a los 37 y $1 / 2$ semanas de gestación, con un peso de $2690 \mathrm{~g}$, producto de primera gestación; presentó además frenillo sublingual.

\section{DISCUSIÓN}

La fibromatosis digital infantil (FDI) es una neoplasia benigna de rara presentación $(4,7,8)$. Fue descrita por primera vez, en 1965, por Reye ${ }^{(2)}$. Laskin y col reunieron, de los archivos del Instituto de Patología de las Fuerzas Armadas de EE UU, 69 lesiones de FDI en 57 pacientes $^{(1)}$.

Esta entidad presenta tres características que lo diferencian de los demás fibromas: 1) presentación limitada a los dedos de manos y pies en niños; 2) marcada tendencia a la recurrencia; y, 3) hallazgo en el estudio anatomopatológico de cuerpos de inclusión citoplasmáticos ${ }^{(1-4,6,6,7,16)}$.

La lesión produce nódulos en forma de cúpula en los dedos; rara vez se afectan los índices y pulgares ${ }^{(7)}$. Son lesiones únicas o múltiples, congénitas o no; son raras en la infancia tardía o la vida adulta. La recidiva y la persistencia se dan con frecuencia ${ }^{(17)}$, así como también pueden regresionar espontáneamente. Se desconoce su etiología; se sospechó que existía una etiología viral, pero estudios diversos la han desechado ${ }^{(7)}$. Así mismo, se ha encontrado lesiones extradigitales con cuerpos de inclusión similar a la FDI, como en un tumor filodes de mama y otras ${ }^{(1,9-12)}$,

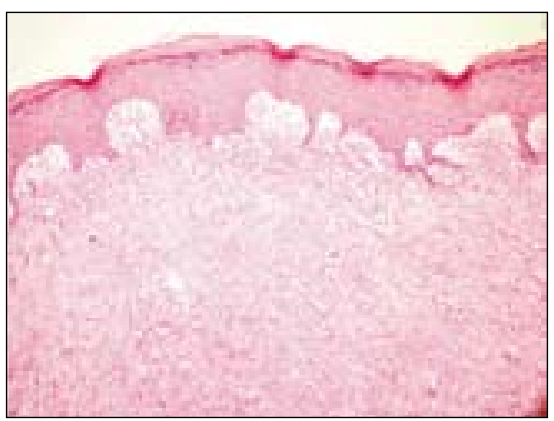

Figura 2. Histología de la tumoración con hematoxilina-eosina.

e igualmente fibromas digitales infantiles sin la presencia de cuerpos de inclusión ${ }^{(1)}$.

Histopatológicamente, es un tumor que no presenta cápsula y se extiende por la dermis y tejido celular subcutáneo. Está conformado por haces entrelazados de células fusiformes en un fondo de colágeno. Los núcleos son ovales, vesiculares. En el citoplasma de las células se hallan cuerpos de inclusión esféricas, eosinofílicas, del tamaño de los eritrocitos, de disposición paranuclear; se tiñen de rojo con la coloración tricrómica de Masson y son ácido paraamino salicílico (PAS) negativos. A la inmunohistoquímica, estas células expresan vimentina, actina músculo liso especifica, desmina, CD99, CD117 ${ }^{(1)}$. A la microscopía electrónica son miofibroblastos y se ha postulado que los cuerpos de inclusión son productos de degradación de los miofilamentos de actina, aunque se discute esto último $^{(7)}$.

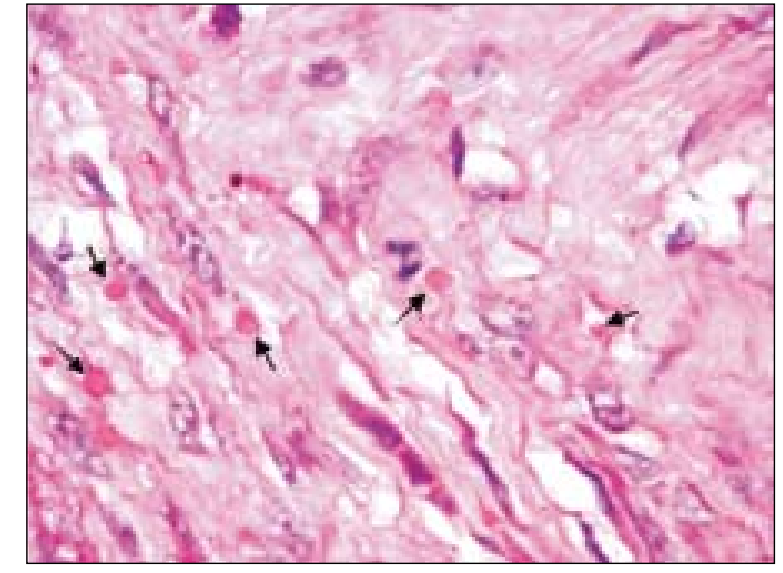

Figura 3. Haces de tejido formados por células fusiformes.

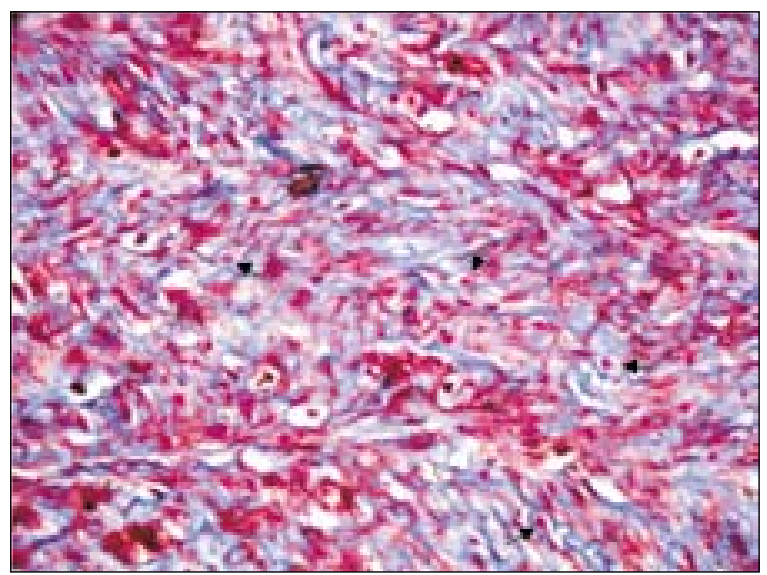

Figura 4. Coloración tricómica de Masson. 


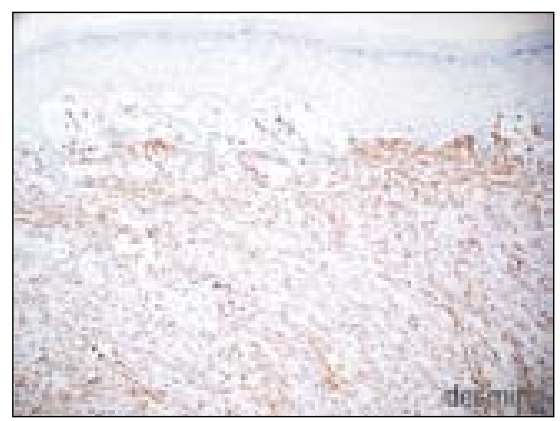

Figura 5. Coloración con desmina.

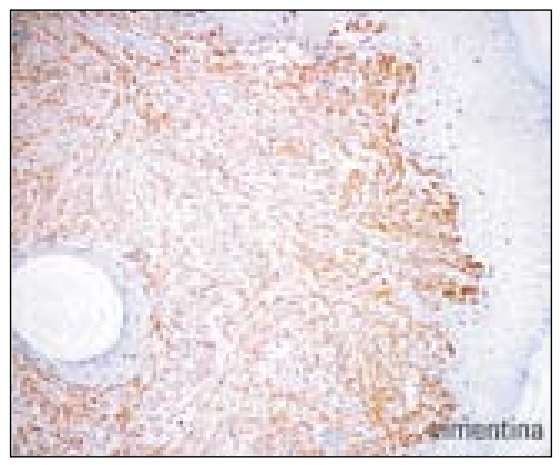

Figura 6. Coloración con vimentina.

En resumen, se presenta un clásico caso de FDI congénito, de acuerdo a la literatura publicada ${ }^{(1-3,8,18)}$. En el fibroma digital infantil o fibroma por cuerpos de inclusión, el cuadro clínico y las características patológicas son características, pero existen aspectos que todavía quedan para estudio porque no están totalmente aclarados y que Laskin ${ }^{(1)}$ las resume y expone con brillante raciocinio, como son: la naturaleza y etiopatogenia, el reporte de casos con presencia de cuerpos de inclusión similares a la FDI en lesiones extra digitales y el manejo clínico controversial; en tanto, se ha informado la regresión espontánea en algunos casos ${ }^{(13,14)}$. Algunos autores recomiendan la observación de la lesión posterior a una biopsia, en lugar de su extirpación, u otros tratamientos alternativos $^{(15)}$.

\section{REFERENCIAS BIBLIOGRÁFICAS}

1. Laskin WB, Miettinen M, Fetsch JF. Infantile digital fibroma/ fibromatosis. A clinicopathologic and immunohistochemical study of 69 tumors from 57 patients with long-term follow-up. Am J Surg Pathol. 2009;33:1-13.

2. Reye RDK. Recurring digital fibrous tumors of childhood. Arch Pathol. 1965;80:228-336.

3. De León B, Fernández JM. Fibromatosis con cuerpos de inclusión (fibromatosis digital infantil) en un niño. An Med Asoc Med Hosp ABC. 2004;49(3):147-50.

4. Stiller D, Katenkamp D. Morphogenesis of intracytoplasmic dense (inclusion) bodies in a recurring digital fibrous tumor of childhood. Light and electronic-microscopic investigations. Virchows Arch Path Anat Histol. 1975;367:73-81.

5. Failla V, Wauters O, Nikkels-Tassoudji N, Carlier A André J, Nikkels AF. Congenital infantile digital fibromatosis: a case report and review of the literature. Rare Tumors. 2009;1:73-4.

6. Gilber-Barness E. Potter's. Pahology of the Fetus, Infant and Child. Second edition. Elsevier Publishers. 2010:1721.

7. Weedon D. Patologia de la Piel. Ed Marban. 2002:765-6.

8. Taylor HO, Gellis SE, Schmidt BA, Upton J, Rogers GF. Infantile digital fibromatosis. Ann Plast Surg. 2008;61(4):472-6.
9. Hiraoka N, Mukai M, Hosoda Y, Hata J. Phyllodes tumor of the breast containing the intracytoplasmic inclusion bodies identical with infantile digital fibromatosis. Am J Surg Pathol. 1994;18(5):506-11.

10. Ortega E, Aranda FI, Chuliá MT, Niveiro M, Payá A, Segui J. Phyllodes tumor of the breast with actin inclusions in stromal cells: diagnosis by fine-needle aspiration cytology. Diagn Cytopathol. 2001;25(2):115-7.

11. Canioni D, Richard S, Rambaud C, Manach Y, Nezelof C. Lingual localization of an inclusion body fibromatosis (Reye's tumor). Pathol Res Pract. 1991;187(7):886-9;

12. Viale G, Doglioni C, Iuzzolino P, Bontempini L, Colombi R, Coggi G, Dell'Orto P. Infantile digital fibromatosis-like tumour (inclusion body fibromatosis) of adulthood: report of two cases with ultrastructural and immunocytochemical findings. Histopathol. 1988;12(4):415-24.

13. Kawaguchi M, Mitsuhashi Y, Hozumi Y, Kondo S. A case of infantile digital fibromatosis with spontaneous regression. J Dermatol. 1998;25(8):523-6.

14. Ishii N, Matsui K, Ichiyama S, Takahashi Y, Nakajima $\mathrm{H}$. A case of infantile digital fibromatosis showing spontaneous regression. $\mathrm{Br} \mathrm{J}$ Dermatol. 1989;121(1):129-33.

15. Guadagnini DE, Durango A, Perich L. Fibromatosis digital infantil (reporte de un caso tratado con crioterapia). Dermatol Venezolana. 1995;33(3).

16. Sungur N, Kilinç H, Ozdemir R, Sensöz O, Ulusoy MG. Infantile digital fibromatosis: an unusual localization. J Pediatr Surg. 2001;36(10):1587-9.

17. Kawabata H, Masada K, Aoki Y, Ono K. Infantile digital fibromatosis after web construction in syndactyly. J Hand Surg Am. 1986;11(5):741-3.

18. Kartings M, Hicks M.J, Levy ML. Hipertrofias dérmicas. En: Wolff Klauss, Ed. Dermatologia en

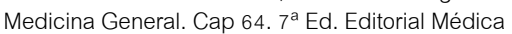
Panamericana SA. 2009:551.

Trabajo recibido el 29 de noviembre de 2010 y aceptado para publicación el 22 de diciembre de 2010.

Correo electrónico: neryromero@hotmail.com 